Stephanie Krüger ${ }^{1}$ Peter Bräunig ${ }^{2}$

\section{Eisschmelze an Nord- und Südpol infolge der globalen Erwärmung}

Polar Ice Caps Melting as a Result of the Gobal Warming
Wenn das Ihre Definition einer bipolaren Störung ist, dann sollten Sie unbedingt dieses Sonderheft lesen. Aber auch, wenn Sie nicht zu den über 1000 Befragten gehören, die in einer Studie von Angermeyer u. Matschinger [1] angaben, die bipolare Störung sei eine Manifestation des Treibhauseffektes, birgt dieses Heft eine Menge interessanter Informationen über die Diagnostik und Therapie der akuten Manie und manisch-depressiver Mischzustände. Beides wird immer komplexer, was zum einen an dem enormen Kenntniszuwachs der letzten Jahre, insbesondere in Bezug auf die Diagnostik manischer Episoden liegt, zum anderen aber auch an dem sich ständig vergrößernden therapeutischen Spektrum, das es ermöglicht, dieser Komplexität durch spezifische Psychopharmaka und störungsspezifische Psychotherapien gerecht zu werden. Dieses Sonderheft gibt allen Berufsgruppen, die sich mit bipolaren Störungen befassen, einen Überblick über die wesentlichen Aspekte moderner Maniediagnostik, Pharmako- und Psychotherapie, einschließlich Psychoedukation.

Der erste Beitrag dieses Heftes stellt therapeutische Leitlinien, entwickelt von der World Federation of the Societies of Biological Psychiatry vor, die dabei helfen können, Manien nach einem Algorithmus zu therapieren. Im zweiten Beitrag kommt das Bipolar Selbsthilfe Netzwerk zu Wort: hier wird erstmals auf die Bedürfnisse bipolarer Patienten in Bezug auf ihre stationäre Behandlung aufmerksam gemacht; ein Aspekt, der in unserem klinischen Alltag leider viel zu kurz kommt, der aber enorme Auswirkungen auf die Zufriedenheit und damit auch auf die Compliance unserer bipolaren Patienten hat.
Danach folgen Beiträge über zugelassene atypische Neuroleptika in der Maniebehandlung - diese Substanzen spielen eine immer größere Rolle in der Therapie bipolarer Störungen allgemein und manischer Episoden im Besonderen. Ein weiteres Thema in diesem Heft ist die Darstellung der schwierigen Therapie manischdepressiver Mischzustände. Diese Episoden sind im klinischen Alltag vor allem auf Akutstationen weitaus häufiger als die euphorischen „Lehrbuchmanien“, werden aber oftmals verkannt und fehldiagnostiziert. Die daran anschließenden Beiträge befassen sich mit der Diagnostik von Manien im Kindes- und Jugendalter, mit neuropsychologischen Testverfahren bei bipolaren Störungen und mit Selbstbeurteilungsinstrumenten zur Diagnostik von Manien.

Die Schwerpunkte der letzten Beiträge sind die pharmakologische Rezidivprophylaxe und die Psychotherapie bipolarer Störungen. Letztere ist in Form einer spezifischen Verhaltenstherapie und Psychoedukation unabdingbar für die moderne Behandlung der bipolaren Erkrankung.

Wir hoffen, dass wir mit diesem Sonderheft das Eis brechen und eine globale Erwärmung auslösen können: bei Ihnen, für die bipolare Störung und für die Belange derjenigen, die von ihr betroffen sind.

\section{Literatur}

${ }^{1}$ Angermeyer MC, Matschinger H. Was ist eine bipolare Störung? Psychiat Prax 2005; 32: 289-291 